\title{
Understanding the essence of life
}

\author{
Ruiyu Zhu*1 \\ *Jiangnan University, Wuxi, P.R. China.
}

\begin{abstract}
Absract
What is the essence of life? Everyone may have a different answer in their hearts. However, is there a most basic answer to the nature of life? To respond, it is necessary to be able to remove everything at the material level and place it into the process of natural evolution. In this article, I present a systematic analysis of the root of life for the first time. First, I propose that the movement form of life is a layer-by-layer iteration of "change and unchanged." Moreover, the existing form of life is a periodic cycle of five elements. Second, initiative is the first driving force of life. Whether for individuals or society, we need to be able to actively construct in order to truly unleash the vitality of our lives. Finally, human life lies in the construction of a conscious environment full of vitality. Human life for society resides in the ability to encourage of more human lives to bloom. Although it seems that one should be able to take the initiative to control everything, happiness cannot be separated from a material base. Humans are merely a more advanced form of natural evolution-a form that is able to actively regulate natural evolution. The most essential truth of life described here is the truth consistent with the driving force of nature. Therefore, the essence of life described here can help everyone's life grow and bloom.
\end{abstract}

Many people ponder the nature of life. So, what is life? Maybe everyone has different answers in their hearts. Yet the true essence of life is found after stripping away the various material foundations that sustain it. Does life itself exist in isolation? Or, is it related to the evolutionary process after the formation of matter? There is no doubt that our physical body is the highest form of matter that evolves after the formation of elementary particles. Moreover, our spirit also derives from our objective world. This has been well-explained in my previous articles. Life itself is connected to the evolution of matter and our conscious world is a more advanced form of evolution.

\section{The bottom of life, the iteration of change and unchanged}

In the 42nd chapter of Tao Te Ching, there is the following: Dao produces one, one produces two, two produces three, and three produces everything. One finds that such words remain relevant even following the advent of science. Their resonance stems from the similarity in iteration. Laozi discovered the most basic rule of things from a relatively macro perspective. What exactly does this "one" mean? Does it mean energy? If it refers to energy, then "two" are the yin and yang, the unity of change and unchanged. Yin means relatively unchanged, while Yang means relatively changed.

\footnotetext{
${ }^{1}$ This is a working document which has not been peer reviewed yet. Please address any comment or remark to ry_zhu@hotmail.com or ry_zhu@sina.com.
} 
At a higher level, this "one" is a big change, and "two" is a big "unchanged." Change represents a bottom layer; it provides energy for the development of everything. The unchanged however, provides existence and constraints for the development of everything. The constraints on change are manifest in the existence of shape and space-time. The dynamic balance between change and unchanged results in the evolution of higher dimensions; they become three.

From another point of view, three refers to a higher level of change. Above three, there is the emergence of individuals and the environment. The beginnings of a complex system emerge. This also is the basis for the existence of things. The development of things to a higher level is the basis for the formation of everything and the existence of life phenomena. Any existence comes from changes at a lower level. It then forms a balance with the environment to obtain relative stability, albeit under various constraints. This constraint or environment-driven balance is "unchanged." If there is a drive for further development, it is based on a higher level of change. Such changes are based on higher-level original things and various changes resulting from "unchanged" iterations. Such various changes also may be referred to as the life bloom of original "unchanged."

From this understanding, emerges the idea that the most essential truth of life should be evolution or iteration based on change and unchanged. All true needs stem from one for vitality. Vitality is not the same as change because it depends upon "unchanged." That "unchanged" becomes the basis for higher-level changes that, in turn, generate even higher-level changes, and ultimately form infinite iterations and evolutions. Of course, to describe phenomena as change and unchanged, only explains the lowest forms; it does not explain a true status quo. What are the basic laws that follow "change and unchanged?" Obviously, we need a type of law that would permit understandings of the constraints on the movement of things, predictions about the direction of movements, and comprehension of the boundaries that impact those movements.

Because unchanged phenomena are easy to grasp, they prove relatively easy to study. That is the focus of modern science. The law of change from one level to another is relatively difficult to grasp. Within a system, the initial stage is the process of transformation to an "unchanged" state. It then moves from a "unchanged" state to a higher "change" state. Therefore, it manifests as a change of state; it is the result of the interaction of internal rules and external environment. The state can be divided into five parts that reflect the Five Elements theory: metal, water, wood, fire, and earth. The essence of these five elements is to clarify the process of information on display in the material world. ${ }^{2}$

The dynamic of the five elements are embedded in all complex systems. If the information processed by metal is life information, the five states referred to by the five elements also are the five development stages of life: metal refers to the stage of pregnancy and basic structure formation of the new system; water refers to the stage where the new system obtains energy and basic substances from the environment; wood refers to the stage of higher material formation shaped by information from metal; fire refers to the stage characterized by function and high-level matter that responds to the metal information; and earth refers to the collapse of the system's

\footnotetext{
${ }^{2}$ I have provided detailed scientific explanations of the Five Elements theory in another article.
} 
structure and feedback to metal. The process of these five elements makes the complex system exhibit life cycle phenomena. From the perspective of the life cycle, the five stages involved in the five elements can be called pregnancy, infancy, development, adulthood, and old age.

It may be said that everything in a complex system exists in different specific cycles. This period is derived from the periodicity of the system in which a being is engaged. In order to determine the measure of time, it is necessary to identify it's periodic position. For higher latitude time periods, this position is more important. Only when the personal cycle is synchronized with the environmental cycle and the social cycle at a higher latitude can we stimulate greater vitality.

\section{Human life}

Everyone has two selves; one is material and the other is spiritual. The physical self gradually evolved from a fertilized egg to a mature individual. This self continues to age and eventually leave this world. The spiritual self is derived from a reflection of the environment experienced. That self may grow but be less affected by age. Spiritually, I can cross time and space and exist in various ways. From the display of physiological information in the material world, we can see that human lives can be divided into metals (fertilized eggs to pre-birth), water (infancy), wood (juvenile growth to maturity), fire (height no longer increases ), and earth (loss of reproductive ability until death). Obviously, in the metal stage, the differentiation of various organs of the human body is completed. The body then enters the environment independently to absorb matter and energy. The water stage is a process of learning and acquiring basic viability, a period when it begins to actively obtain food and energy from the environment. The wood phase is the process characterized by rapid development until completion. In this process, the most important thing is sexual maturity. This process may display information about human life. The fire stage is the process in which function is on display, characterized by the maintenance of social functioning and bearing children. Earth is the process of material return, accumulation, and the transmission of life information.

After the period of sexual maturity, the human body ceases to physically grow. However, the growth of human mental faculties may continue throughout one's life. The latest research shows that brain cells of a person who is 90 years old remain plastic. Human beings come from fertilized eggs. The appearances of human beings change from childhood to old age, as their cells change. With the development of science, various human organs also can be replaced by artificial organs. Yet "I" itself has not changed following organ replacement. Human nature is not physiological, but spiritual. People are determined by the connections among their neural networks. The connections among these neural networks and the continuous division of hierarchies form the basis of consciousness. This represents the fundamental reason why humans are superior to animals. There are two cognitive models, one is perceptual cognition and the other is rational cognition. The main manifestation of perceptual cognition is to incorporate the changing elements of "I" into the cognitive process. Perceptual cognition is restricted by the body itself and cannot grow independently and indefinitely. Rational cognition is a kind of cognition that excludes "I" and can develop deeper. Rational cognition is a growth structure. The entire cognitive growth process is undoubtedly a dynamic process of constant iteration and exchange of determinism and non-determinism. Certainty exists in the brain and is the basis for 
further development or architecture. Uncertainty is an integral part of cognitive growth and provides multiple possibilities for growth. Rational cognition is the foundation on which the spiritual I may grow and the foundation for the spiritual I to possess greater vitality.

\section{The purpose of life is to build a conscious environment with vitality}

What is the purpose of living? Perhaps everyone has their own answer. In a previous paper, I already discussed the construction mechanism of consciousness and its influence on further cognition. It is true that one carries out a range of activities, but we only can experience what is in the context of consciousness. The purpose of our cognitive activities is simply to make the conscious environment converge with the objective real world, so that it reflects the objective world. However, due to differences in the physical environment, life cycle, and internal state of each person, it is not possible to truly reflect the objective world. The reflection of the objective world itself is an interaction. That interaction cannot be separated from either side of the movement. The objective material world may change slowly, but people's changes cause interactions that change and promote cognitive changes. The objective material world itself is constantly changing. Therefore, it is impossible to fully understand. Consequently, one needs to hold open one's state of consciousness. Openness enables a better understanding of the world. The only thing that can be conscious or experienced is the conscious environment in the human brain. Often the conscious world will be rendered by emotions and fall into traps. When this happens, the conscious world appears to be very different from the real world and mental problems may ensue. Consciousness itself has a demand for expansion, which is why most people like new things. This could explain why we experience inner release when we contemplate open views, such as those from on top of mountains, the sea, or a virtual open scene.

Due to the influence of culture and personal senses, humans often unconsciously trap consciousness in a state of relative rigidity and lack of vitality. This lack of vitality and conservative state further makes actions lose vitality. The willingness to try again is impeded by the memory of bad experiences or ingrained perspectives. One loses the courage to try again due to frustration. Alternatively, one fears rejection by others. One risks the loss of true understanding of self through an overestimation of the others' opinions. True growth is inner growth. Essence is a spiritual self. We need to build a mentally viable self and establish corresponding viable conscious environments. Only by creating a conscious environment with vitality will behaviors characterized by vitality appear.

\section{Initiative is the first driving force of life}

As I stated above, evolution overrides the laws of matter. It is evolution that promotes the formation of laws of matter. The formation of laws of matter ensures evolution. I also proposed that the entire process of the five elements is actually one characterized by the movement between information and material presentation, followed by feedback from the material presentation that generates incremental changes in the existing information. The power of evolution comes from the system itself. The system creates an individual from the environment. The essence of this individual is a closed system isolated from the environment. The first step is the generation and distribution of relevant information that enables the system to be constructed. The 
second is the use of that information to actively obtain the required energy and basic materials from the environment. The third step is the generation of related structures.The fourth step is the display of functions. Finally, there is a regression process. If there is no iteration after the regression, it is difficult to achieve an increase in information and growth at a higher level. The initiative is characterized by selection, the process of energy consumption, and the original driving force. We may observe the process from seed germination to plant formation. First, the seeds need to obtain the necessary water and nutrients from the environment, which is an active intake process. There are many seeds and plants in the soil. We cannot imagine that the environment will actively transport water and nutrients to the seeds or plants.

The initiative actually may be divided into two distinct types; one is characterized by internal construction and the other by external pursuits. In the real world, we often treat initiative as pursuits rather than as internal construction, which creates many problems. The natural power of man is from the inside out. Material is guided by the spirit. That is the material basis of our existence. However, for the inner self, because it is the basis of consciousness and the inner top-down driving force of all behaviors, it is hard to see and rarely recognized or understood. Similarly, the spiritual world is a system that needs to be constantly rebuilt in order for it to regain vitality. Without that continual reconstruction, the spiritual world would be bound by the external environment, hostage to desire and physical existence, and a mere servant of the flesh. Initiative is very important, and this can be reflected in the real world. Almost all successful people in real society are more proactive in behavior. However, the real problem is that these more active people are often passive in their spiritual construction. After their basic objectives are achieved, they show a loss of vitality and become imprisoned by physical desires or existing ideologies. The most important reason for this is the lack of vitality in the spiritual world. Humans need to be able to actively build the inner value of life. Only in this way can the true value and meaning of life be obtained and life directions and rhythms unified with the process of natural evolution. Initiative also is the first driving force for human growth. There is a need for students to build the energy of initiative. The development of society demands people's increased participation, as opposed to the participation of a few who are selected through social conventions. We need to let students take the initiative to care about the country and society and let people take the initiative to care about the weak and the social issues that impact them.

\section{Human society needs an active construction of the spirit}

Throughout the entire history of human society, the establishment of social organizations has been accompanied by shared ideal goals. New ruling classes were formed by people's support of those groups. However, after the formation of the ruling class, society often returned to its original problems, with little growth. The root of the problem is that people only pursue the satisfaction of needs; they rarely think about how such needs are presented and whether the current solution is reasonable. Humans have proved unable to reflect on and carry out active creation at the inner spiritual or cognitive levels. Therefore, the material world has been actively transformed for improved human survival, but the inner spiritual world does not seem to be much different from thousands of years ago. The primitive nature of the spiritual world makes it difficult for humans to greatly improve society's overall index of happiness. Rather, they remain constrained by the original spiritual world, filled with 
contradictions, and lacking of true vitality. Therefore, human beings need to break away from a passive reception of signals from the natural environment and concepts pertaining to the social environment, and move towards the path of active construction. The purpose of reconstruction is to make the spiritual world grow and fill with vitality. There is a need to return various laws or principles to their original positions and turn the underlying laws into the basic principles that guide their lives. The goal is to make the spiritual structure conform to the laws of natural evolution, in order to let the beauty and vitality of life blossom.

\section{Life's meaning is to have people's lives blossom}

What is the purpose and meaning of life? For a fruit tree that bears many fruits, the fruit attracts more creatures and allow these creatures to survive. However, the fruit tree itself does not enjoy the tastes. Fruit trees have a strong vitality because of growth, not because of how much they have consumed. Human beings actually live in a conscious environment that they shape. This conscious environment is the world itself on which we may focus. Life in the conscious environment is what we feel, what we recognize, and what we expect. Only when things in the conscious environment have vitality can we better feel the existence of life. The purpose of life is to contribute to the blossoming of more people's lives; only when more people's lives bloom, will society improve. Beautiful beliefs will continue to iterate and spread, bearing beautiful fruits in human society. This is a self-fulfilling self, a "spiritually viable me." It is a self that makes my spiritual world broader, more prosperous, more vital, and truly full of love. This definition of the purpose of life is in line with the natural driving force because the natural driving force of people is from inside to outside; the natural driving force of society or groups moves from individuals to groups. In fact, only by defining life in this way can we truly conform to the laws of personal development and social growth. The selection of talents with similar standards can make the organizational structure conform to the trend of natural forces. Because the selection criteria includes the goal of having more people's lives blossom, the selected people will begin by naturally following the direction of social development and take the ability to help subordinates blossom. They will not only enable people who are capable of using their abilities and adopting their views, but also other people who promote overall development. If one only uses ability as the selection criterion, they often find that the talents selected prioritize the continuation of their own views and capabilities; others are used only to pursue those same goals or reproduce those same perspectives. In this sense, most people are unable to truly deploy their abilities and it is difficult to achieve overall growth. Everyone is in a unique time and space. It proves impossible to see all aspects of the problem. Naturally, no matter how capable or intelligent they are, there will be moments when things are not clear or well thought out. In this case, the development of the entire team or organization will inevitably encounter difficulties. Therefore, the definition of life with the direction of social development and the goal of helping others to blossom, is in line with nature's driving force. There exists a deeper meaning. Perhaps it is to restore life to its natural and original meaning. 


\section{References}

1. zhu, ruiyu. "Scientific Connotation of the Five Elements Theory." SocArXiv, 19 June 2020. Web. 